\title{
Características clínico epidemiologicas de los pacientes con Leucemia Aguda del Servicio de Hematologia del Hospital Almanzor Aguinaga Asenjo
}

\author{
Ana María Polo-Capuñay', Carlos Alberto León-Seminario', Joan Flaubert Pérez-Villena', Jhonny David Yovera-Merino', \\ Omar Barraza-Chavesta', Víctor Torres-Anaya', Cristian Díaz-Vélez ${ }^{2}$
}

\section{RESUMEN:}

Objetivo: Identificar las características clínico-epidemiológicas de los pacientes con leucemia en el área de Hematología del H.A.A.A.

Material y Métodos: Estudio retrospectivo, transversal y descriptivo. Se revisaron 55 historias clínicas de pacientes con diagnóstico de Leucemia Aguda, tomadas de la oficina de registro del HNAAA, durante el periodo de Enero 2005 a Marzo 2010.

Resultados: En el periodo de estudio, se diagnosticaron 142 casos de Leucemia, 105 (73.94\%) correspondieron a Leucemia aguda. De ellos, 39 fueron varones y 16 mujeres. El grupo etario más frecuente fue de 31 a 55 años (30.9\%), y el menos frecuente de 0 a 2 años (3.6\%). 38 pacientes (69.1\%) fueron diagnosticados por consulta externa y 17(30.9\%) por emergencia. La leucemia aguda linfoide fue más frecuente en varones, que en mujeres. El 7.27\% de leucemias agudas fue de tipo indeterminado. Dentro de las manifestaciones iníciales recolectadas encontramos en mayor cantidad anemia, palidez, fiebre, malestar corporal, otros (gingivorragia, dolor abdominal y vómitos), en menor cantidad encontramos astenia, fatiga y hemorragia. En cuanto a datos de laboratorio, 20 pacientes (69.1\%) presentaron leucopenia; 33 pacientes $(98.2 \%)$ trombocitopenia y 48 pacientes $(87.3 \%)$ hemoglobina baja.

Conclusiones: La prevalencia de leucemia aguda fue de $73.94 \%$. El grupo etáreo que predominó fue entre 31 a 55 años. Los signos y síntomas más comunes desarrollados durante la enfermedad fueron; esplenomegalia, hepatomegalia, anemia, equimosis y petequias. (Horiz Med 2014; 14(1): 18-23)

Palabras clave: Leucemia mieloide aguda (LMA), Leucemia linfoide aguda (LLA), oncología (Fuente: DeCS BIREME)

\section{Epidemiologic clinical features of patients with acute Leukemia Hematology Hospital Service Almanzor Aguinaga Asenjo}

\section{ABSTRACT}

Objective: To identify the organisms that conform the normal flora of the external auditory canal.

Material and Methods: Retrospective, transversal and descriptive. We reviewed a total of 55 medical records with a diagnosis of acute leukemia, taken from the HNAAA registration office during the period from January 2005 to March 2010.

Results: During the study period, 142 cases were diagnosed with Leukemia, 105 (73.94\%) were acute leukemia. Of the 105 cases, 55 were evaluated. Of these 39 were males and 16 females. The most common age group was between 31-55 years $(30.9 \%)$ and the least frequent was $0-2$ years (3.6\%). 38 patients $(69.1 \%)$ were diagnosed as outpatients and $17(30.9 \%)$ diagnosed in emergency. Acute lymphocytic leukemia is more common in men than in women. The type of acute leukemia was undetermined for $7.27 \%$. Within the initial manifestations collected we found more anemia, pallor, fever, body aches, and others (gingival, abdominal pain and vomiting), whereas we found fewer asthenia, fatigue, and bleeding. For laboratory data, 20 patients $(69.1 \%)$ had leukopenia, 33 patients $(98.2 \%)$ had thrombocytopenia and 48 patients (87.3\%) presented low hemoglobin.

Conclusions: The prevalence of acute leukemia was $73.94 \%$. The predominant age group was between $31-55$ years. The most common signs and symptoms developed during the disease were, splenomegaly, hepatomegaly, anemia, bruising and petechiae. (Horiz Med 2014; 14(1): 18-23)

Key words: Acute myeloid leukemia (AML), acute lymphoblastic leukemia (ALL), oncology. (source: MeSH NLM)

\footnotetext{
1 Estudiantes de la Facultad de Medicina Humana de la Universidad San Martin de Porres - Filial Norte. Chiclayo.

2 Docente del curso de Metodología de la Investigación. Universidad de San Martin de Porres - Filial Norte. Chiclayo.

3 Epidemiólogo clínico. Oficina de Inteligencia Sanitaria del Hospital Nacional Almanzor Aguinaga Asenjo
} 


\section{INTRODUCCIÓN}

El origen de cáncer a la sangre es hasta el momento desconocido, sin embargo, hay evidencia consistente de tres factores:

a) Radiaciones ionizantes.

b) Agentes alquilantes usados en la quimioterapia.

c) Exposición al benceno.

La leucemia, fue el primer cáncer en vincularse a las radiaciones ionizantes después de los bombardeos atómicos en Hiroshima y Nagasaki.

Entre otros factores de riesgo tenemos: Virus oncogenes (retrovirus, HTLV-I), factores genéticos y congénitos, fármacos mielotóxicos (fenilbutazona, cloranfenicol y citostáticos), fumadores.

Las alteraciones que contribuyen a la transformación leucémica de las células hematopoyéticas madres alteran los procesos regulatorios, y se aumenta de forma ilimitada la capacidad de autorrenovación, lo que produce una pérdida del control de la proliferación normal, bloqueo en la diferenciación y resistencia a la muerte celular programada $(1,2,3)$.

Tomando en cuenta solo a la leucemia en su forma aguda, esta afecta a cualquier tipo de pacientes tanto niños, jóvenes como adultos. Sus características clínicas son particulares para este tipo de enfermedad así como sus características epidemiológicas que van a variar de grupo en grupo de personas.

En el Hospital Escuela Dr. Roberto Calderón Gutiérrez (HDRG), se realizó un estudio descriptivoretrospectivo durante el período de Enero 2001 al 2004, cuyo objetivo fue, determinar el comportamiento clínico de las leucemias agudas diagnosticadas en el Servicio de Medicina Interna de dicho hospital.

Entre los resultados, se vió que la edad promedio de los pacientes fue de 33,9 años, pertenecientes al sexo masculino en su mayoría $(58,72 \%)$, ocupación predominante de agricultores y amas de casa, procedentes de área rural en el $63,3 \%$ del total.
Tomando como referencia datos epidemiológicosoncológicos de los hospitales de EsSalud, del total de consultas realizadas, el $1 \%$ corresponde al Servicio de Oncología. Para el año 2007, se estimó una tasa de incidencia de cáncer de 89,3 x 100 000 y una tasa de mortalidad de 49,4 x 100000 asegurados. Es por todo ello que para el año 2011, se nombra al cáncer como una prioridad sanitaria con carácter de urgencia (4).

Un promedio de 1,500 casos nuevos de leucemia aguda se diagnostican cada año en el Perú, y de ese total, un tercio corresponde a la población infantil, según Jorge León Chong, Jefe del Departamento de Oncología Médica del Instituto Nacional de Enfermedades Neoplásicas (INEN).

En el estudio realizado en el Hospital Nacional Almanzor Aguinaga Asenjo en el Departamento de Hemato-Oncología. Se clasificó a los pacientes como grupo de riesgo alto o estándar, según los factores de riesgo (5).

Frecuentemente, la leucemia linfocítica en adultos, se presenta con elevada cuenta leucocitaria, linfoadenopatía, esplenomegalia y curso agresivo de la enfermedad, con compromiso en el sistema nervioso central.

En los niños, este tipo de leucemia es la más común en EEUU, principalmente en el sexo masculino (6).

En un estudio realizado por el Instituto de Hematología e Inmunología publicado en la Revista Cubana de Hematología e Inmunología, se dan a conocer las siguientes características: Insuficiencia medular, debido a la diferenciación o maduración aberrante de células leucémicas, que se acumulan en la cavidad de la medula ósea y desplazan las células hemáticas normales (7).

La finalidad del presente estudio, fue conocer las características de la enfermedad, pues en nuestra ciudad, no se cuenta con estudios específicos ni epidemiológicos de esta entidad. 


\section{MATERIAL Y MÉTODOS}

El estudio fue descriptivo, observacional y transversal.

Se revisaron las historias clínicas de los pacientes diagnosticados de leucemia aguda del área de Hematología del Hospital Nacional Almanzor Aguinaga Asenjo, desde Enero 2005 a Marzo 2010.

El tamaño de muestra fue calculado, teniendo en cuenta un nivel de confianza del $95 \%$, con un error de $5 \%$ y una proporción de $8 \%$, según reporte de datos anteriores (2), con lo cual se obtuvo un tamaño muestral de 55 pacientes.

Se consideró los datos de las Historias Clínicas, de las personas vivas, así como las fallecidas que presentaron la enfermedad en dicho periodo.

Se cumplió con las Normas de ética y no se divulgó ningún dato de los pacientes.

\section{RESULTADOS}

De Enero 2005 a Marzo 2010, se diagnosticaron 142 casos de Leucemia, 105 (73.94\%) correspondieron a Leucemia aguda. Siendo evaluados 55 casos.

Dentro de las características epidemiológicas, el 70.9\% fueron de sexo masculino. Figura 1.

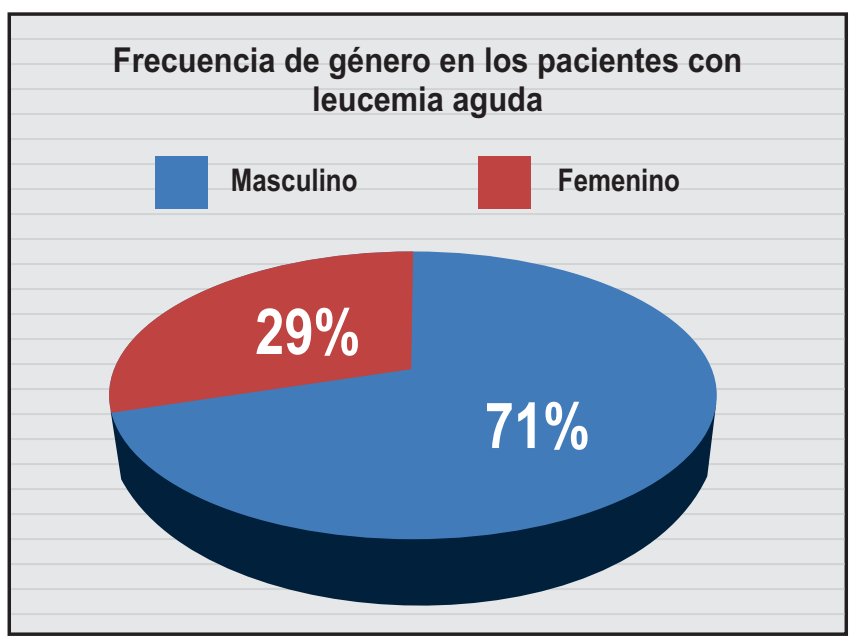

Figura 1. Sexo de los pacientes con leucemia aguda en el Servicio de Hematología del Hospital Nacional Almanzor Aguinaga Asenjo. Enero 2005-Marzo 2010.
El grupo etario predominante fue de 31 a 55 años, y el de menor frecuencia fue de 0 a 2 años. Relacionando el grupo etario con el tipo de leucemia, se observó que en los intervalos de 2 a 9 y de 31 a 35 años, fue más frecuente el tipo mieloide, mientras que entre los 16 a 30 y 31 a 35 años, predominó el tipo linfoide. Los pacientes con leucemia no determinada se hallaron en el intervalo de 56 a 75 años. Figura 2.

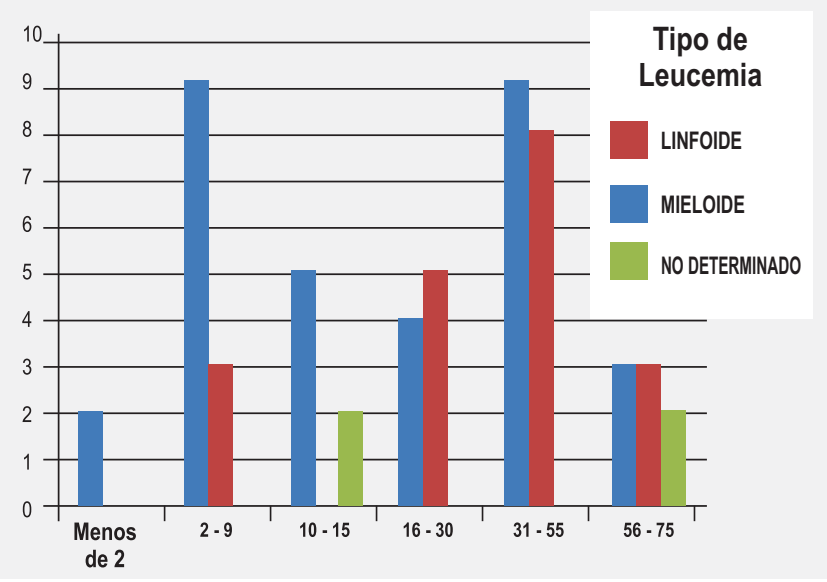

Figura 2. Relación de la edad del paciente y tipo de leucemia de los pacientes con leucemia aguda del Servicio de Hematología del Hospital Nacional Almanzor Aguinaga Asenjo. Enero 2005-Marzo 2010.

Entre las características clínicas, como manifestaciones iniciales predominantes, se observó: palidez, fiebre, malestar corporal, siendo poco frecuente: astenia, fatiga y hemorragia. Los signos y síntomas que se presentaron en el transcurso de la enfermedad fueron: esplenomegalia, hepatomegalia, anemia, equimosis y petequias. Tabla 1.

Los hallazgos de laboratorio fueron: leucopenia, trombocitopenia y hemoglobina baja, con un $69.1 \%$, $98.2 \%$ y $87.3 \%$, respectivamente. Tabla 2 .

\section{DISCUSIÓN}

De todos los casos de leucemia registrados en el periodo de enero del 2005 a marzo del 2010, la mayoría padeció de leucemia aguda (73.94\%).

Predominó el tipo mieloide, linfoide y un bajo porcentaje de no determinado, en éste último tipo 
Tabla 1. Relación entre presentación inicial, sintomatología en el curso de la enfermedad y el tipo de leucemia presente en los pacientes con leucemia aguda en el Servicio de Hematología del HN A. Aguinaga Asenjo. Enero 2005-Marzo 2010.

\begin{tabular}{|c|c|c|c|c|}
\hline & \multicolumn{4}{|c|}{ TIPO DE LEUCEMIA DEL PACIENTE } \\
\hline & LINFOIDE & MIELOIDE & $\begin{array}{c}\text { NO } \\
\text { DETERMINADO }\end{array}$ & TOTAL \\
\hline \multicolumn{5}{|c|}{ PRESENTACIÓN INICIAL } \\
\hline $\begin{array}{l}\text { Malestar } \\
\text { corporal }\end{array}$ & $\begin{array}{c}4 \\
(26.67 \%)\end{array}$ & $\begin{array}{c}9 \\
(60 \%)\end{array}$ & $\begin{array}{c}2 \\
(13.33 \%)\end{array}$ & $\begin{array}{c}15 \\
(27.27 \%)\end{array}$ \\
\hline Sd. anémico & $\begin{array}{c}27 \\
(56.25 \%)\end{array}$ & $\begin{array}{c}17 \\
(35.41 \%)\end{array}$ & $\begin{array}{c}4 \\
(13.33 \%)\end{array}$ & $\begin{array}{c}48 \\
(27.27 \%)\end{array}$ \\
\hline Sd. febril & $\begin{array}{c}20 \\
(64.51 \%)\end{array}$ & $\begin{array}{c}10 \\
(32.25 \%)\end{array}$ & $\begin{array}{c}1 \\
(3.23 \%)\end{array}$ & $\begin{array}{c}31 \\
(56.36 \%)\end{array}$ \\
\hline Astenia & $\begin{array}{c}7 \\
(7 \%)\end{array}$ & $\begin{array}{c}1 \\
(10 \%)\end{array}$ & $\begin{array}{c}2 \\
(2 \%)\end{array}$ & $\begin{array}{c}10 \\
(18.18 \%)\end{array}$ \\
\hline Palidez & $\begin{array}{c}18 \\
(69.23 \%)\end{array}$ & $\begin{array}{c}6 \\
(23.08 \%)\end{array}$ & $\begin{array}{c}2 \\
(7.69 \%)\end{array}$ & $\begin{array}{c}26 \\
(47.27 \%)\end{array}$ \\
\hline
\end{tabular}

\section{DESARROLLADO CON LA ENFERMEDAD}

\begin{tabular}{|c|c|c|c|c|}
\hline Adenopatías & $\begin{array}{c}4 \\
(57.14 \%)\end{array}$ & $\begin{array}{c}2 \\
(28.57 \%)\end{array}$ & $\begin{array}{c}1 \\
(14.28 \%)\end{array}$ & $\begin{array}{c}7 \\
(12.72 \%)\end{array}$ \\
\hline Esplenomegalia & $\begin{array}{c}7 \\
(70 \%)\end{array}$ & $\begin{array}{c}2 \\
(20 \%)\end{array}$ & $\begin{array}{c}1 \\
(10 \%)\end{array}$ & $\begin{array}{c}10 \\
(18.18 \%)\end{array}$ \\
\hline Hepatomegalia & $\begin{array}{c}8 \\
(66.67 \%)\end{array}$ & $\begin{array}{c}3 \\
(25 \%)\end{array}$ & $\begin{array}{c}1 \\
(8.33 \%)\end{array}$ & $\begin{array}{c}12 \\
(21.82 \%)\end{array}$ \\
\hline Anemia & $\begin{array}{c}22 \\
(62.85 \%)\end{array}$ & $\begin{array}{c}11 \\
(31.43 \%)\end{array}$ & $\begin{array}{c}2 \\
(5.71 \%)\end{array}$ & $\begin{array}{c}35 \\
(63.63 \%)\end{array}$ \\
\hline Equimosis & $\begin{array}{c}5 \\
(50 \%)\end{array}$ & $\begin{array}{c}4 \\
(40 \%)\end{array}$ & $\begin{array}{c}1 \\
(10 \%)\end{array}$ & $\begin{array}{c}10 \\
(18.18 \%)\end{array}$ \\
\hline Petequias & $\begin{array}{c}7 \\
(70 \%)\end{array}$ & $\begin{array}{c}2 \\
(20 \%)\end{array}$ & $\begin{array}{c}1 \\
(10 \%)\end{array}$ & $\begin{array}{c}10 \\
(18.18 \%)\end{array}$ \\
\hline
\end{tabular}

Tabla 2. Valores de Laboratorio según cantidad de leucocitos, plaquetas y hemoglobina en los pacientes con leucemia aguda en el Servicio de Hematología del Hospital Nacional Almanzor Aguinaga Asenjo. Enero 2005-Marzo 2010.

\begin{tabular}{|c|c|c|}
\hline & FRECUENCIA & PORCENTAJE \\
\hline \multicolumn{3}{|l|}{ LEUCITOS } \\
\hline LEUCOPENIA & 20 & $36.40 \%$ \\
\hline NORMAL & 18 & $32.70 \%$ \\
\hline LEUCOCITOS & 17 & $30.90 \%$ \\
\hline \multicolumn{3}{|l|}{ PLAQUETAS } \\
\hline TROMBOCITOPENIA & 33 & $60.00 \%$ \\
\hline NORMAL & 21 & $38.20 \%$ \\
\hline TROMBOCITOSIS & 1 & $1.80 \%$ \\
\hline \multicolumn{3}{|l|}{ HEMOGLOBINA } \\
\hline BAJA & 48 & $87.30 \%$ \\
\hline NORMAL & 6 & $10.90 \%$ \\
\hline ALTA & 1 & $1.80 \%$ \\
\hline
\end{tabular}

se encuentran los pacientes con mayor antigüedad de diagnóstico, debido a que en esos años no se contaba con la tecnología necesaria para la detección adecuada correspondiente al tipo de leucemia a la que pertenecía.

El porcentaje del sexo masculino fue mucho mayor que en pacientes del sexo femenino, este resultado concuerda con todos los estudios tomados como referencia para este estudio.

Entre el grupo etario más frecuente encontramos los pacientes con edades de 31-55 años, y el menos frecuente es de 0-2 años.

Si relacionamosel año genérico y el tipo de leucemia, se halló un mayor porcentaje de pacientes que sufren de leucemia linfoide aguda en los intervalos de 2 a 9 y de 31 a 35 años, mientras que en el tipo de leucemia mieloide, la mayor cantidad se 
presenta en pacientes de 16 a 30 y de 31 a 35 años. Finalmente los pacientes con leucemia no determinada se ubican en el intervalo de 56 a 75 años, que son los pacientes con mayor tiempo de diagnóstico.

Se halló relación estadística significativa $p=0.048$ $(<0.05)$ entre el tipo de leucemia con la edad del paciente.

Entre las manifestaciones clínicas iníciales, tenemos que la mayor parte de pacientes con leucemia linfoide, presentó malestar corporal, anemia, fiebre y palidez, mientras que la fatiga, astenia y hemorragia se presentaron en un bajo porcentaje. En el tipo de leucemia mieloide, los síntomas más frecuentes fueron: malestar corporal y hemorragia.

Estos resultados concuerdan con los datos obtenidos de una de nuestras fuentes bibliográficas que también considera como síntomas principales, los obtenidos en este estudio (6).

Se halló relación estadística significativa $(p=0.015$ > 0.05 ) entre el tipo de leucemia con la manifestación inicial referente a malestar corporal, más no se halló ninguna relación estadística entre el tipo de leucemia con las demás manifestaciones iníciales.

La frecuencia de signos y síntomas desarrollados durante el proceso de la enfermedad, con respecto al tipo de leucemia que se presentó, tenemos que; en la leucemia linfoide hubo predominio de adenopatía, organomegalia, anemia, equimosis y petequias, mientras que en la leucemia mieloide, hubo mayor presencia de hemorragia.

Estos resultados concuerdan con los datos registrados en el texto "Apuntes de cancerología" (6).

No se halló relación estadística significativa $(p>$ 0.05 ) entre el tipo de leucemia con la sintomatología desarrollado por el paciente en el transcurso de su enfermedad.

En el momento del diagnóstico los pacientes, presentaron en forma general, deficiencia de los elementos sanguíneos, como leucocitos, plaquetas, así como también deficiente nivel de hemoglobina, lo cual produj o que se halle un mayor porcentaje de pacientes con anemia como sintomatología inicial.

Gracias a la cantidad de población y muestra, y la disponibilidad de historias clínicas de pacientes que padecían la patología tratada, fue factible el desarrollo del presente estudio, ya que se logró cumplir con los obj etivos y metas trazados.

En conclusión, la leucemia aguda fue más frecuente en varones con predominio de malestar corporal, palidez y fiebre. Con hepatomegalia, anemia, equimosis y petequias. Predominando los niveles bajos de leucocitos, plaquetas y hemoglobina.

\section{Fuentes de financiamiento}

El estudio ha sido autofinanciado por los autores.

\section{Conflicto de interés:}

Los autores declaran no tener ningún conflicto de interés en el trabajo realizado.

\section{REFERENCIAS BIBLIOGRÁFICAS}

1. Margolin J, Steuber P, Poplack D. Acute Iymphoblastic leukemia. Principles and Practices of Pediatric Oncology. Fifth edition. Philadelphia; 2006; 538 - 90.

2. Golub TR, Arceci RJ. Acute myelogenous leukemia. Principles and Practices of Pediatric Oncology. Fifth edition Philadelphia; 2006; 591 - 644.

3. Pui Ch H. Acute lymphoblastic leukemia. In: Ching-Hon Pui editor. Childhood leukaemia. Second edition. Ed. Memphis Tennessee, USA: St. Jude Children's Research Hospital; 2006; 439-472. 
4. Garcia P, Cotrina G, Arauco P, Untama J.¿Es factible el trasplante autólogo de progenitores hematopoyéticos en el Hospital Nacional Almanzor Aguinaga Asenjo, Chiclayo? [Carta al editor]. Rev Cuerpo Méd HNAAA 2012; 5(3).

5. Echevarria CP, Gonzales ML. Estudio Descriptivo de Factores asociados al pronóstico de Vida de los pacientes con Leucemia Linfocitica Aguda. (Tesis Médico Cirujano). Chiclayo, Universidad Nacional Pedro Ruiz Gallo; 1993.

6. Solidoro A. Apuntes de Cancerología, Segunda edición. Ed. Hozlo, Lima - Perú, 2005; 481-

503.

7. Milanés $M$, Losada $R$, Hernández $P$, Agramonte O, Rosell E. Aspectos clínicos y epidemiológicos de la leucemia mieloide aguda en el anciano. Rev Cubana Hematol Inmunol Hemoter [revista en la Internet]. 2002 Abr [citado 2010 Abril 17]; 18(1):Disponible en: <http://scielo.sld.cu/ scielo. php?script=sciarttext\&pid=\$0864028920 02000100002\&lng=es>

\section{Correspondencia:}

Ana María Polo-Capuñay

Dirección: Filial Norte USMP. Chiclayo, Perú 